\title{
Electron-Scale Quadrants of the Hall Magnetic Field Observed by the Magnetospheric Multiscale spacecraft during Asymmetric Reconnection
}

\author{
Rongsheng Wang, ${ }^{1,2, *}$ Rumi Nakamura, ${ }^{2}$ Quanming Lu, ${ }^{1, \dagger}$ Wolfgang Baumjohann, ${ }^{2}$ R. E. Ergun, ${ }^{3}$ \\ J. L. Burch, ${ }^{4}$ Martin Volwerk, ${ }^{2}$ Ali Varsani, ${ }^{2}$ Takuma Nakamura, ${ }^{2}$ Walter Gonzalez, \\ Barbara Giles, ${ }^{6}$ Dan Gershman, ${ }^{6}$ and Shui Wang ${ }^{1}$ \\ ${ }^{1}$ Key Laboratory of Geospace Environment, Department of Geophysics and Planetary Science, \\ University of Science and Technology of China, Hefei 230026, China \\ ${ }^{2}$ Space Research Institute, Austrian Academy of Sciences, Graz 8042, Austria \\ ${ }^{3}$ Department of Astrophysical and Planetary Sciences, University of Colorado Boulder, Boulder, Colorado 80303, USA \\ ${ }^{4}$ Southwest Research Institute, San Antonio, Texas 78238, USA \\ ${ }^{5}$ Instituto Nacional de Pesquisas Espaciais, Sao Jose dos Campos 12227-010, Brazil \\ ${ }^{6}$ NASA, Goddard Space Flight Center, Greenbelt, Maryland 20771, USA
}

(Received 17 January 2017; published 25 April 2017)

\begin{abstract}
An in situ measurement at the magnetopause shows that the quadrupole pattern of the Hall magnetic field, which is commonly observed in a symmetric reconnection, is still evident in an asymmetric component reconnection, but the two quadrants adjacent to the magnetosphere are strongly compressed into the electron scale and the widths of the remaining two quadrants are still ion scale. The bipolar Hall electric field pattern generally created in a symmetric reconnection is replaced by a unipolar electric field within the electron-scale quadrants. Furthermore, it is concluded that the spacecraft directly passed through the inner electron diffusion region based on the violation of the electron frozen-in condition, the energy dissipation, and the slippage between the electron flow and the magnetic field. Within the inner electron diffusion region, magnetic energy was released and accumulated simultaneously, and it was accumulated in the perpendicular directions while dissipated in the parallel direction. The localized thinning of the current sheet accounts for the energy accumulation in a reconnection.
\end{abstract}

DOI: 10.1103/PhysRevLett.118.175101

Magnetic reconnection, as an effective mechanism for converting magnetic energy into plasma kinetic and thermal energy, has been believed to be responsible for a large number of explosive phenomena in space, astrophysics, and laboratory plasmas [1,2]. Because of the Hall effect, ions are unmagnetized and electrons are magnetized in the ion diffusion region [3,4], and the relative motion between ions and electrons results in a quadrupole pattern of the outof-plane magnetic field and a bipolar pattern of the Hall electric field pointing to the center at both sides of the reconnecting current sheet [5-14]. The unique signature of the Hall quadrupolar structure has now been regarded as one critical criterion to identify the ion diffusion region $[9,10,15-18]$. The quadrupolar structure is symmetric in an antiparallel reconnection (i.e., the shear angle $\alpha=180^{\circ}$ ) and can be distorted by a guide field $\left(\alpha<180^{\circ}\right)$ [19,20]. It is well known that the width of each quadrant is of the ion scale [3-6,15]. If the asymmetric distribution of the reconnecting field and plasma was considered, the quadrupole pattern was replaced by a bipolar pattern, as predicted in simulations [21,22]. However, a recent measurement shows that the quadrupole pattern might still exist in an asymmetric reconnection [23].

In this Letter, we present an asymmetric magnetic reconnection event encountered at the magnetopause by the Magnetospheric Multiscale (MMS) spacecraft, which was designed to explore electron physics in a reconnection [24]. Since the MMS crossed the ion diffusion region both north and south of the $X$ line, all four Hall quadrants can be measured very well. Such a situation should be frequently found from the MMS measurement, based on the preliminary analysis.

The data from several instruments are used. The mission and its instruments are described in several papers [24-28]. During 06:04:40-06:06:00 UT, on 22 October 2015, the MMS was located at $[9.6,3.5,-2.0] R_{E}$ in Geomagnetic Solar Magnetospheric coordinates. At this time, the spacecraft traversed the magnetopause outbound, with a short excursion back to the magnetosphere in 06:05:16-06:05:21 UT. Figure 1 represents an overview of the crossing in the local boundary normal LMN coordinates (see Supplemental Material [29]).

In the beginning, the spacecraft was located in the magnetosphere, characterized by the stable and northward $B_{L}$ [ $\sim 58 \mathrm{nT}$, Fig. 1(a)], low density [ $<2 \mathrm{~cm}^{-3}$, Fig. 1(h)], and high-energy electrons [ $>2 \mathrm{keV}$, Fig. 1(i)]. After 06:05:03 UT, the MMS entered into the magnetosheath, with higher density $\left(10 \mathrm{~cm}^{-3}\right)$ and low-energy electrons $(<1 \mathrm{keV})$. From 0605:05 to 0605:32 UT, high-speed ion flows $\left(v_{i L}\right)$ up to $150 \mathrm{~km} / \mathrm{s}$ were detected, comparable 


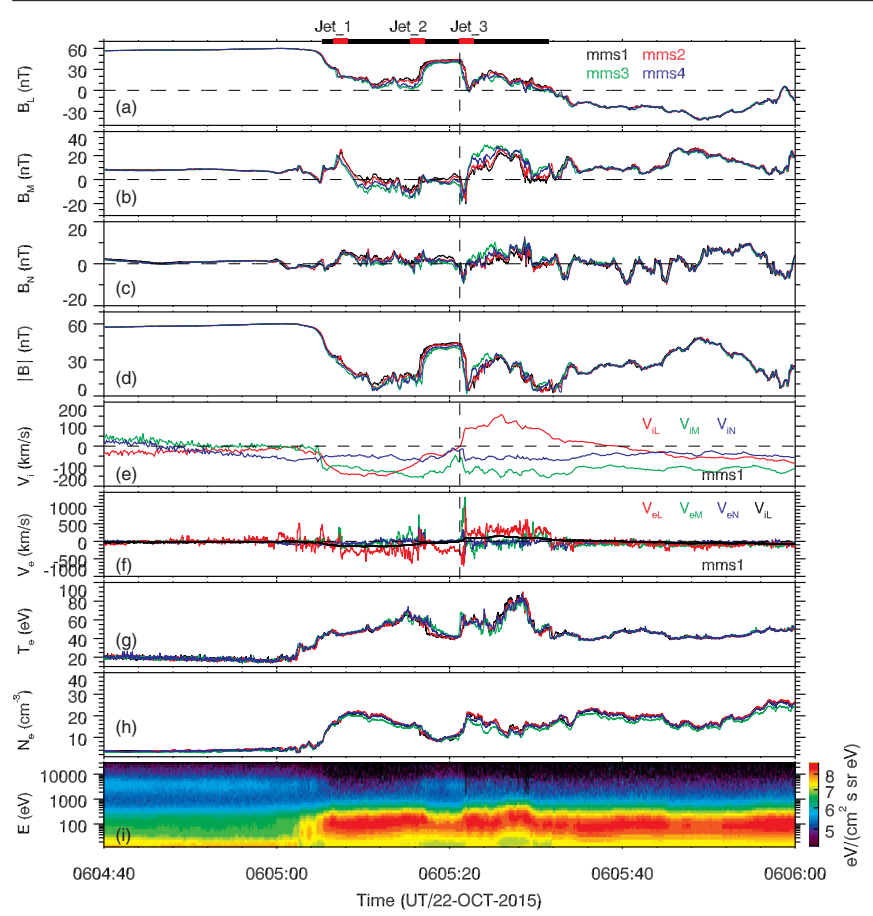

FIG. 1. (a)-(d) $\mathrm{B}_{L}, \mathrm{~B}_{M}, \mathrm{~B}_{N}$, and $|\mathbf{B}|$ at the four satellites, (e) ion flows $v_{i L}, v_{i M}$, and $v_{i N}$, and (f) electron flows $v_{e L}, v_{e M}, v_{e N}$, and $v_{i L}$ at mms1. The electron (g) temperature, (h) density, and (i) energy spectrum at mms1.

to the magnetosheath Alfvén speed $v_{A}=146 \mathrm{~km} / \mathrm{s}$ $\left(N \approx 20 \mathrm{~cm}^{-3}\right.$ and $\left.B_{L} \approx 30 \mathrm{nT}\right)$. The ion flow was initially southward and then reversed to northward at $\sim 0605: 21.3$ UT (the vertical dashed line), accompanied with the $B_{N}$ reversal from Sunward to anti-Sunward [Fig. 1(c)]. Comparing $v_{e L}$ and $v_{i L}$ [Fig. 1(f)], $v_{e L}$ was much higher than $v_{i L}$ during 0605:06-0605:32 UT (the black bar at the top). The decoupling of the ion and electron flows represents a feature of the ion diffusion region, consistent with the violation of the ion frozen-in condition (not shown). So, we conclude that the MMS passed through the ion diffusion region of an asymmetric reconnection. The ambient field $\left(B_{M}\right)$ was $\sim 10$ nT before 06:05:06 UT [Fig. 1(b)] and disturbed around the reconnection site. The ratio of the guide field to the reconnecting field was only $\sim 0.2$; therefore, the guide field was weak. Based on the observations, the MMS trajectory is shown in Fig. 2(a).

In the southward ion flows, $B_{M}$ was substantially enhanced for $\sim 1.0 \mathrm{~s}$ from $10 \mathrm{nT}$ at $\sim 0605: 06.5 \mathrm{UT}$ to $25 \mathrm{nT}$ at $\sim 0605: 07.5 \mathrm{UT}$ and then became negative for $\sim 12 \mathrm{~s}$ during 0605:08-0605:20 UT [Fig. 1(b)]. In the northward flows, $B_{M}$ was mainly positive except a short negative spike $(\sim 0.8 \mathrm{~s})$ at $\sim 0605: 21.5 \mathrm{UT}$. Given the trajectory, it seems that a strongly distorted quadrupolar structure was observed. The durations of the lower-right and upper-right quadrants were approximate $0.8 \mathrm{~s}$, much shorter than their counterquadrants in the magnetosheath ( $\sim 9$ s). At 0605:32 UT when the MMS left the ion diffusion region north of the $X$ line into the magnetosheath, a southward electron flow was measured [Fig. 1(f)], corresponding to the inflowing Hall electrons.

Just after the $v_{i L}$ reversal point, an intense electron jet pulse [Fig. 1(f)] was detected at $B_{L} \sim 30 \mathrm{nT}$, marked as Jet_3, while the MMS was moving towards the magnetosheath. The jet pulse was located immediately north of the $X$ line and was enlarged in the first column in Fig. 3. The electron jets were simultaneously observed in the $L$ and $M$ directions [Figs. 3(a) and 3(b)]. Their speed was as high as $1300 \mathrm{~km} / \mathrm{s}$, substantially larger than the Alfvén speed $v_{A}(146 \mathrm{~km} / \mathrm{s})$ yet less than the electron Alfvén speed in the magnetosheath $\left(v_{e, A}=6200 \mathrm{~km} / \mathrm{s}\right)$. The electron shear flow was evident in the $L$ direction, and $v_{e L}$ evolved from south to north [Fig. 3(a)]. $v_{e M}$ peaked near the center of the shear flow at each satellite [Fig. 3(b)]. Furthermore, the jet pulse was directly related to the negative spike of $B_{M}$ [Figs. 1(b) and 3(e)], i.e., the upper-right quadrant [Fig. 2(a)]. $v_{e N}$ was much weaker than the other two components [Fig. 3(c)] and also changed sign from Sunward $\left(v_{e N}>0\right)$ to anti-Sunward $\left(v_{e N}<0\right)$. Since Jet_3 was measured in close proximity to the $X$ line, the $v_{e N}$ reversal indicates that the spacecraft encountered the stagnation point, as proposed in simulations [30].

In Ref. [31], Jet_3 was interpreted as a filamentary current associated with a reconnection, as many other currents in the exhaust far away from the $X$ line. In order to establish the relation of the jet to the electron diffusion region, we further analyzed the similar jet pulses south of the $X$ line at $\sim 0605: 17$ UT and $\sim 0605: 07$ UT [Fig. 1(f)], shown in the second (Jet_2) and third (Jet_1) columns in Fig. 3, respectively. Jet_1 and Jet_2 were both detected at $B_{L} \sim 25$ nT. Jet_1 was detected while the MMS was approaching the magnetosheath [Fig. 3(t)], and Jet_2
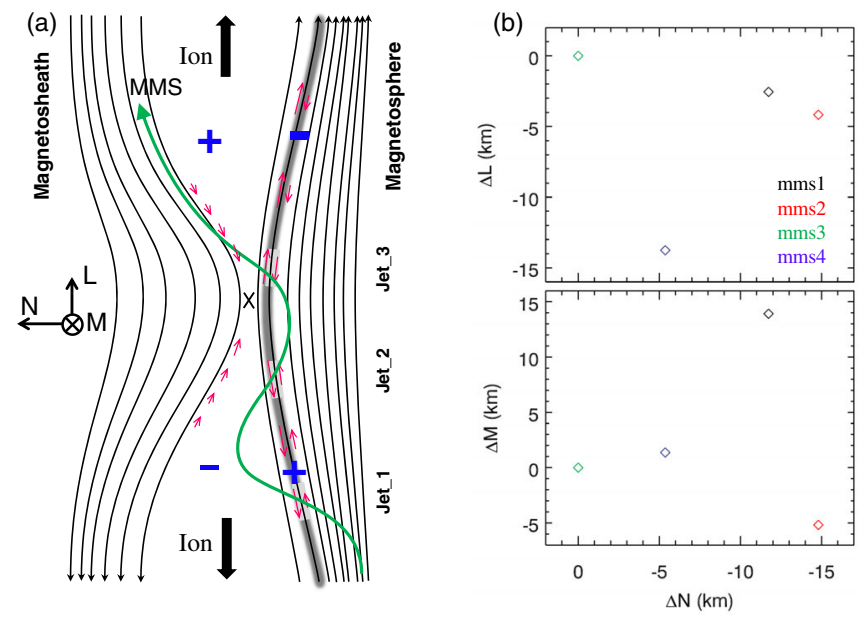

FIG. 2. (a) A schematic illustration for the reconnection. The shadow region denotes the electron current layer, and the pink arrows mean the electron flows. The blue signs of "+" and "-" mean the positive and negative $\mathrm{B}_{M}$. (b) Relative position of the four satellites at 06:05:21 UT. 


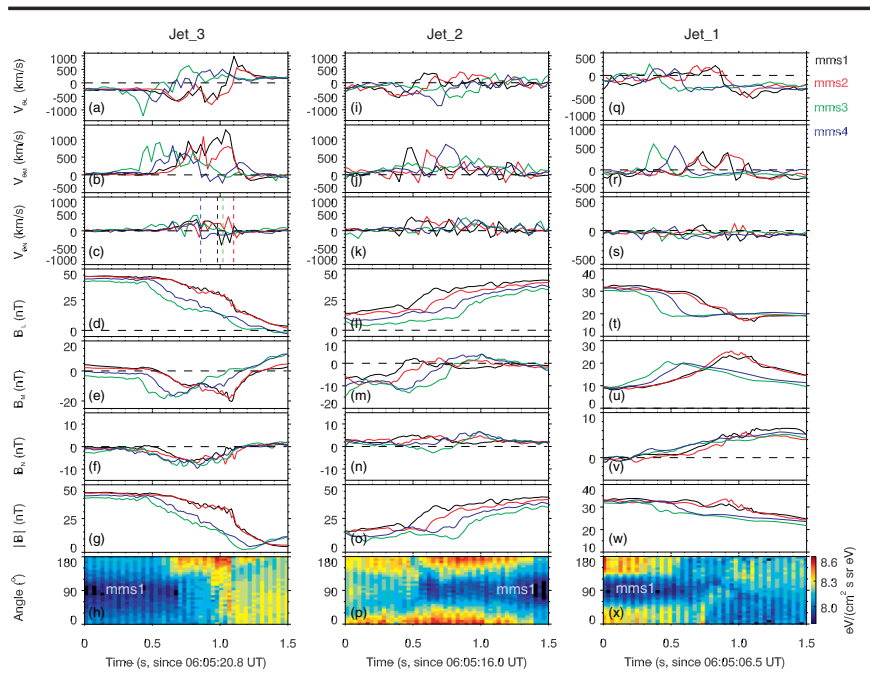

FIG. 3. (a)-(c) $v_{e L}, v_{e M}$, and $v_{e N}$ at all four satellites, (d)-(g) $\mathrm{B}_{L}, \mathrm{~B}_{M}, \mathrm{~B}_{N}$, and $|\mathbf{B}|$, and (h) electron pitch angle distribution during 200-2000 eV at mms1. Panels (i)-(p) and (q)-(x) show the data in Jet_2 and Jet_1, respectively.

was measured as the MMS was moving deeply into the magnetosphere [Fig. 3(1)]. $v_{e L}$ changed its direction from north to south [Fig. 3(q)] in Jet_1 while reversed from south to north [Fig. 3(i)] in Jet_2. In Jet_1 and Jet_2, the speed of the southward jet was considerably stronger than the northward jet, and $v_{e M}$, pointing to the dawn side, got its maximum value within the shear flow [Figs. 3(j) and 3(r)]. In Jet_1, $B_{M}$ was significantly enhanced from $\sim 10$ to $25 \mathrm{nT}\left[\delta B_{M} \sim 15 \mathrm{nT}\right.$, Fig. $\left.3(\mathrm{u})\right]$. In Jet_2, $B_{M}$ was positive also within the shear flow and $\delta B_{M}$ was close to $15 \mathrm{nT}$ yet without a clear peak [Fig. $3(\mathrm{~m})]$. The positive enhancements of $B_{M}$ in Jet_1 and Jet_2 corresponded to the lower-right quadrant of the quadrupole pattern.

Combining all three jet pulses, a dawnward out-of-plane electron jet $\left(v_{e M}\right)$ was always observed at $B_{L} \sim 25 \mathrm{nT}$ and bounded by an electron shear flow $v_{e L}$. The corresponding currents were shown in Figs. 4(b)-4(d), 4(j)-4(l), and $4(\mathrm{r})-4(\mathrm{t})$, respectively. For simplicity, only the data at mms1 were shown. Based on the total current $\mathbf{J}=$ $n q\left(\mathbf{v}_{\mathrm{i}}-\mathbf{v}_{\mathrm{e}}\right)$ (thick traces) and electron current $\mathbf{J}=-n e \mathbf{v}_{\mathrm{e}}$ (thin traces) in Figs. 4(b), 4(j), and 4(r), the deviation between the total current and electron current is small, indicating that the current was mainly carried by electrons. Apparently, there was an electron current layer on the magnetosphere side of the ion diffusion region. The electron current layer consisted of an out-of-plane current $\left(j_{M}\right)$ near its center and the bidirectional current $\left(j_{L}\right)$ at its boundaries [Figs. 4(c), 4(k), and 4(s)]. The out-ofplane current mainly contributed to the perpendicular current, and the bidirectional current formed the fieldaligned current [Figs. 4(d), 4(l), and 4(t)], which can be found also in the electron pitch angel distribution [Figs. 3(h) and 3(x)] except for in Jet_2 [Fig. 3(p)]. The out-of-plane current layer was in accordance with simulations of an asymmetric reconnection [21,22,32], where an out-of-plane current layer was produced by the electric field drift $(\mathbf{E} \times \mathbf{B})_{M}$.

The current layer was mainly lying in the $L-M$ plane, and its normal velocity was $-34.4,17.5$, and $-27.1 \mathrm{~km} / \mathrm{s}$ for Jet_3-Jet_1, respectively (see Supplemental Material [29]). As for Jet_3, its thickness was $\sim 8.6 c / \omega_{p e}$ (electron inertial length) at mms 3 and mms 4 and became $\sim 5.8 c / \omega_{p e}$ at mms1 and mms2. The layer thicknesses in Jet_2 and Jet_1 were $\sim 2.9$ and $\sim 4.5 c / \omega_{p e}$, respectively. So, the current layer (the corresponding quadrants of Hall field) was electron scale. However, the width of the counterquadrant was estimated to be 4.3 ion inertial lengths $\left(\Delta t \sim 9 \mathrm{~s}, 0605: 23-0605: 32 \mathrm{UT} ; v_{N} \sim-34.4 \mathrm{~km} / \mathrm{s}\right)$.

Because of the intermittent presence of cold plasma wakes from the MMS [33], the electric fields here were recalibrated to remove baseline offsets of $\mathbf{E}+\mathbf{V}_{e} \times \mathbf{B}$ that endured for more than $5 \mathrm{~s}$. This offset removal does not affect a deviation between $\mathbf{E}$ and $-\mathbf{V}_{e} \times \mathbf{B}$ with a duration of less than $2.5 \mathrm{~s}$. Prior to 0605:21.4, an abrupt cold plasma wake, seen in the ion distribution and in strong plasma waves [34], exceeds $2 \mathrm{mV} / \mathrm{m}$ in $E_{z}$. In the first $0.6 \mathrm{~s}$ of the Jet_3 interval, the uncertainty in $E_{z}$ is $\sim 5 \mathrm{mV} / \mathrm{m}$, which primarily affects the $L$ component. In each jet pulse, the striking signature was the intense positive $E_{N}^{\prime}\left(\mathbf{E}^{\prime}=\mathbf{E}+\mathbf{V}_{\text {xline }} \times \mathbf{B}\right)$ in the $X$-line frame [blue traces in Figs. 4(e), 4(m), and 4(u)], where $\mathbf{V}_{\text {xline }}$ is the velocity of the $X$-line frame relative to the MMS. $E_{N}^{\prime}$ directed to the magnetosheath and was as intense as $30 \mathrm{mV} / \mathrm{m}$ at Jet_3, up to $10 \mathrm{mV} / \mathrm{m}$ at Jet_2 and less than $10 \mathrm{mV} / \mathrm{m}$ at Jet_1. $v_{e M}$ decreased with the distance from the $X$ line $[\sim 1300 \mathrm{~km} / \mathrm{s}$ at Jet_3, $\sim 900 \mathrm{~km} / \mathrm{s}$ at Jet_2, and $\sim 400 \mathrm{~km} / \mathrm{s}$ at Jet_1 in Figs. 4(a), 4(i), and 4(q), respectively], and so did the current density $j_{M}\left[2.0 \mu \mathrm{A} / \mathrm{m}^{2}\right.$ at Jet_3, $1.2 \mu \mathrm{A} / \mathrm{m}^{2}$ at Jet_2, and $0.8 \mu \mathrm{A} / \mathrm{m}^{2}$ at Jet_1 in Figs. 4(c), 4(k), and 4(s), respectively]. $j_{M}$ and $v_{e M}$ show a similar evolution to $E_{N}^{\prime}$, suggesting that they were formed by the $\left(\mathbf{E}^{\prime} \times \mathbf{B}\right)_{M}$ drift. The calculated drift velocity $\left[\mathbf{E}^{\prime} \times \mathbf{B} /|\mathbf{B}|^{2}\right.$, thick traces in Figs. 4(f), 4(n), and 4(v)] matched pretty well with the measured electron velocity ( $\mathbf{V}_{e \perp}$, thin traces) except in the $M$ component at Jet_3 and Jet_2. In particular, a larger difference can be found in the $M$ component at Jet_3, which indicates that the electrons were slipping from the magnetic field.

The electron shear flow was another distinct feather in the layer. In the north of the $X$ line (Jet_3), the electrons were moving southward, i.e., towards the $X$ line (inflowing electrons) and on the magnetospheric side and northward, i.e., away from the $X$ line (outflowing electrons), on the other side. In the south of the $X$ line (Jet_1 and Jet_2), on the contrary, electrons were flowing northward on the magnetospheric side and southward on the other side. This shear flow was consistent with the observed $B_{M}$, which was negative north of the $X$ line and positive south of the $X$ line. The comparable inflowing and outflowing speeds at Jet_3 indicate that Jet_ 3 should be very close to the $X$ line. 

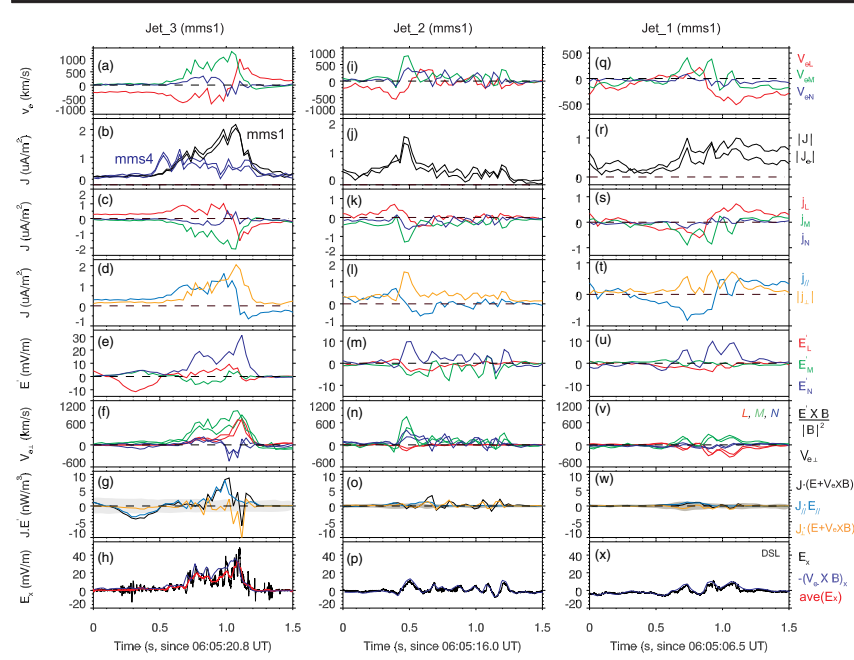

FIG. 4. (a) $v_{e L}, v_{e M}$, and $v_{e N}$ at mms1, (b) the total (thick curve) and electron current (thin curve) density, (c) $j_{L}, j_{M}$, and $j_{N}$, (d) $j_{\|}$ (blue curve) and $\left|j_{\perp}\right|$ (yellow curve), (e) the electric field ( $E_{N}^{\prime}$, blue curve; $E_{M}^{\prime}$, red curve; $E_{L}^{\prime}$, green curve) in the $X$-line frame $\mathbf{E}^{\prime}=\mathbf{E}+\mathbf{V}_{\text {xline }} \times \mathbf{B}$, (f) the drift velocity $\left(\mathbf{E}^{\prime} \times \mathbf{B} /|\mathbf{B}|^{2}\right)$ and $\mathbf{V}_{\perp}=\left[\mathbf{B} \times(\mathbf{E} \times \mathbf{B}) /|\mathbf{B}|^{2}\right],(\mathrm{g}) \mathbf{J} \cdot\left(\mathbf{E}+\mathbf{V}_{e} \times \mathbf{B}\right)$ (black curve), $\mathbf{J}_{\|} \cdot \mathbf{E}_{\|}$(pale blue curve), $\mathbf{J}_{\perp} \cdot\left(\mathbf{E}+\mathbf{V}_{e} \times \mathbf{B}\right)_{\perp}$ (yellow curve), the shadow means the errors of $\mathbf{J} \cdot\left(\mathbf{E}+\mathbf{V}_{e} \times \mathbf{B}\right)$, and (h) $E_{x}$ (black curve) and $-\left(v_{e} \times B\right)_{x}$ (blue curve). Panels (i)-(p) and (q)-(x) show the data in Jet_2 and Jet_1, respectively.

In Figs. 4(h), 4(p), and 4(x), the DSL (Despun, Sunpointing L-momentum vector) coordinates where the electric field was measured was used. In Jet_1 and Jet_2, $E_{x}$ and $-\left(v_{e} \times B\right)_{x}$ matched perfectly [Figs. 4(p) and 4(x)], and $\mathbf{J} \cdot\left(\mathbf{E}+\mathbf{V}_{e} \times \mathbf{B}\right)$ was very small [Figs. 4(o) and 4(w)]. Hence, the electrons were frozen-in and the energy dissipation was negligible. In Jet_ $3, E_{x}$ or its average value [the electrostatic fluctuations removed, red trace in Fig. 4(h)] show a clear deviation from $-\left(v_{e} \times B\right)_{x}$, up to $20 \mathrm{mV} / \mathrm{m}$. It means that the electron frozen-in condition was violated. Namely, the MMS entered into the inner electron diffusion region [35,36]. Inside Jet_3, $\mathbf{J}_{\|} \cdot \mathbf{E}_{\|}$was always positive [blue trace in Fig. 4(g)] and $\mathbf{J}_{\perp} \cdot\left(\mathbf{E}+\mathbf{V}_{e} \times \mathbf{B}\right)_{\perp}$ was basically positive except for a $40 \mathrm{~ms}$ interval near the $\left|J_{\perp}\right|$ peak at 06:05:21.9 UT [Fig. 4(d)]. Such a short interval corresponded to a region of $1.0 \mathrm{c} / \omega_{\text {pe }}$, where $\mathbf{J}_{\perp}$. $\left(\mathbf{E}+\mathbf{V}_{e} \times \mathbf{B}\right)_{\perp}$ and $\mathbf{J} \cdot\left(\mathbf{E}+\mathbf{V}_{e} \times \mathbf{B}\right)$ were both negative. Some simulations predicted that the Hall field in an asymmetric reconnection displayed a dipolar pattern $[21,22,32]$. However, evident Hall quadrupolar structure was confirmed in this asymmetric reconnection. The distorted quadrupole pattern in a symmetric reconnection was attributed to the guide field effect $[19,20]$. The guide field deflected the outflow electron jets and caused a distorted pattern. The previous observations showed that their widths were ion scale near the $X$ line; even the ratio of the guide field to the asymptotic field was up to 0.3 [20]. In the present event, the ratio was only $\sim 0.2$, yet the two quadrants adjacent to the magnetosphere were compressed into the electron scale. So, the guide field cannot be the unique reason. It seems that an asymmetric distribution of the field and plasma is the main reason for the enhanced compression.

In Jet_3, the current layer thickness became thinner while mms 2 and mms 1 crossed it. It means that the layer might be thinning. A thinning current layer would cause an increase of the local field and current intensity. The current intensity at mms 1 was substantially stronger than that at mms4 [Fig. 4(b)], and a clear field enhancement was indeed observed at mms 1 and mms 2 , in comparison with the smooth decrease at mms 3 and mms 4 [Fig. 3(g)]. The negative $\mathbf{J}_{\perp} \cdot \mathbf{E}_{\perp}^{\prime}$ and $\mathbf{J} \cdot\left(\mathbf{E}+\mathbf{V}_{e} \times \mathbf{B}\right)$ in Jet_3 could be attributed to the thinning process, since magnetic energy will be accumulated in a thinning current layer. In addition, the two minimums of the southern jet $v_{e L}$ [at $\sim 0605: 21.5$ and 0605:21.7 UT in Fig. 3(a)] at mms1 and mms 2 can be referred to the thinning also. A possible scenario is that, as mms 1 and mms 2 encountered the first $v_{e L}$ peak at 0605:21.5 UT, the layer started to thin and then made the two satellites retreat out of the layer. Approximately $100 \mathrm{~ms}$ later (at 0605:21.6 UT), they got into the layer again and then crossed it. The current sheet thinning has been regarded as one necessary condition for reconnection onset [37]. Our observations indicate that the thinning can take place in an ongoing reconnection, instead of only before the onset. The energy dissipation and accumulation can occur simultaneously near the inner electron diffusion region.

All the MMS data used in this work are available at the MMS science data center [38].

R. W. appreciates the helpful discussion from P. Pritchett, C. T. Russell, and C. Zhao in UCLA and the valuable suggestions from Y. Khotyaintsev in Swedish Institute of Space Physics. R. W. and R. N. sincerely thank the suggestions and comments from T. Phan at University of California, Berkeley. This work is supported by the National Science Foundation of China (NSFC) Grants No. 41674143, No. 41474126, No. 41331067, and No. 41421063) and by the National Basic Research Program of China (2013CBA01503). The work at Austria is supported by the Austrian Science Fund (FWF) No. I2016-N20.

*rswan@ustc.edu.cn †mlu@ustc.edu.cn

[1] R. A. Treumann and W. Baumjohann, Astron. Astrophys. Rev. 23, 4 (2015).

[2] M. Yamada, R. Kulsrud, and H. T. Ji, Rev. Mod. Phys. 82, 603 (2010).

[3] B. U. O. Sonnerup, in Solar System Plasma Physics, edited by L. T. Lanzerotti, C. F. Kennel, and E. N. Parker (North-Holland, New York, 1979), p. 45.

[4] T. Terasawa, Geophys. Res. Lett. 10, 475 (1983).

[5] M. A. Shay, J. F. Drake, R. E. Denton, and D. Biskamp, J. Geophys. Res. 103, 9165 (1998). 
[6] Z. W. Ma and A. Bhattacharjee, Geophys. Res. Lett. 25, 3277 (1998).

[7] M. Oieroset, T. D. Phan, M. Fujimoto, R. P. Lin, and R. P. Lepping, Nature (London) 412, 414 (2001).

[8] X. H. Deng and H. Matsumoto, Nature (London) 410, 557 (2001).

[9] F. S. Mozer, S. D. Bale, and T. D. Phan, Phys. Rev. Lett. 89, 015002 (2002).

[10] A. Vaivads, Y. Khotyaintsev, M. Andre, A. Retino, S. C. Buchert, B. N. Rogers, P. Decreau, G. Paschmann, and T. D. Phan, Phys. Rev. Lett. 93, 105001 (2004).

[11] X. R. Fu, Q. M. Lu, and S. Wang, Phys. Plasmas 13, 012309 (2006).

[12] T. Nagai, I. Shinohara, M. Fujimoto, M. Hoshino, Y. Saito, S. Machida, and T. Mukai, J. Geophys. Res. 106, 25929 (2001).

[13] A. Runov et al., Geophys. Res. Lett. 30, 1579 (2003).

[14] G. Paschmann, Geophys. Res. Lett. 35, L19109 (2008).

[15] W. H. Matthaeus, C. D. Cothran, M. Landreman, and M. R. Brown, Geophys. Res. Lett. 32, L23104 (2005).

[16] Y. Ren, M. Yamada, H. Ji, S. P. Gerhardt, and R. Kulsrud, Phys. Rev. Lett. 101, 085003 (2008).

[17] H. Ji, Y. Ren, M. Yamada, S. Dorfman, W. Daughton, and S. P. Gerhardt, Geophys. Res. Lett. 35, (2008).

[18] J. P. Eastwood, T. D. Phan, M. Oieroset, and M. A. Shay, J. Geophys. Res. 115, A08215 (2010).

[19] J. P. Eastwood, M. A. Shay, T. D. Phan, and M. Oieroset, Phys. Rev. Lett. 104, 205001 (2010).

[20] R. S. Wang et al., J. Geophys. Res. 117, A07223 (2012).

[21] P. L. Pritchett, J. Geophys. Res. 113, A06210 (2008).
[22] M. Shay, T. Phan, C. C. Haggerty, M. Fujimoto, J. F. Drake, K. Malakit, P. Cassak, and M. Swisdak, Geophys. Res. Lett. 43, 4145 (2016).

[23] R. E. Denton, B. U. O. Sonnerup, H. Hasegawa, T. D. Phan, C. T. Russell, R. J. Strangeway, B. L. Giles, and R. B. Torbert, J. Geophys. Res. 121, 9880 (2016).

[24] J. L. Burch, T. E. Moore, R. B. Torbert, and B. L. Giles, Space Sci. Rev. 199, 5 (2016).

[25] C. T. Russell et al., Space Sci. Rev. 199, 189 (2016).

[26] R. E. Ergun et al., Space Sci. Rev. 199, 167 (2016).

[27] P. A. Lindqvist et al., Space Sci. Rev. 199, 137 (2016).

[28] C. Pollock et al., Space Sci. Rev. 199, 331 (2016).

[29] See Supplemental Material at http://link.aps.org/ supplemental/10.1103/PhysRevLett.118.175101 for the local boundary coordinates and the thicknesses of the current layer.

[30] P. A. Cassak and M. A. Shay, Phys. Plasmas 14, 034701 (2007).

[31] T. Phan et al., Geophys. Res. Lett. 43, 6060 (2016).

[32] C. Huang, Q. M. Lu, S. Lu, P. R. Wang, and S. Wang, J. Geophys. Res. 119, 798 (2014).

[33] E. Engwall, A. I. Eriksson, M. Andre, I. Dandouras, G. Paschmann, J. Quinn, and K. Torkar, Geophys. Res. Lett. 33, L06110 (2006).

[34] R. Ergun et al., Geophys. Res. Lett. 43, 5626 (2016).

[35] H. Karimabadi, W. Daughton, and J. Scudder, Geophys. Res. Lett. 34, L13104 (2007).

[36] M. A. Shay, J. F. Drake, and M. Swisdak, Phys. Rev. Lett. 99, 155002 (2007).

[37] N. Singh, Phys. Rev. Lett. 109, 145001 (2012).

[38] See https://lasp.colorado.edu/mms/sdc/. 